\title{
Probing Lorentz symmetry with the Pierre Auger Observatory
}

Denise Boncioli ${ }^{* a}$ for the Pierre Auger Collaboration ${ }^{b}$

${ }^{a}$ DESY, Platanenallee 6, 15738 Zeuthen, Germany

${ }^{b}$ Observatorio Pierre Auger, Av. San Martín Norte 304, 5613 Malargüe, Argentina

E-mail: auger_spokespersons@fnal.gov

Full author list: http://www.auger.org/archive/authors_icrc_2017.html

Effects of Lorentz invariance violation (LIV) can be present at energies much lower than the quantum gravity scale. Here the possible LIV effects in ultra-high energy cosmic rays (UHECRs) are investigated. To this aim, modifications of the propagation of UHECR protons and nuclei in the extragalactic space due to LIV effects are taken into account. For the first time a fit of both flux and composition of UHECRs as measured by the Pierre Auger Observatory is used in order to evaluate the constraining power of current data on LIV parameters.

35th International Cosmic Ray Conference - ICRC2017

10-20 July, 2017

Bexco, Busan, Korea

${ }^{*}$ Speaker. 


\section{Introduction}

One of the fundamental symmetries of relativity is Lorentz Invariance (LI). In the last decades there has been progress in testing deviations from LI. A unified theory of Quantum Mechanics, that describes the universe at small scales, and General Relativity, that describes it at large scales, is still unknown. Lorentz Invariance Violations (LIV) are expected to be observable, if any, at energies near the Planck scale $\left(M_{\mathrm{Pl}} \sim 1.2 \times 10^{28} \mathrm{eV}\right)$. If compared with the current maximal energy attainable in accelerators, the observation of such phenomena requires particles to have 15 orders of magnitude larger than the LHC center-of-mass energy.

High energy astrophysics provides opportunities to probe energies much larger than the accelerator ones. In particular, in this work we study the interactions of Ultra-High-Energy Cosmic Rays (UHECRs), meaning with that particles that hit the Earth's atmosphere with energies above $10^{18}$ $\mathrm{eV}$. Such particles are mainly expected to have extragalactic origin, since they arrive nearly isotropically to the Earth and the Galactic magnetic field would not be able to confine them. Moreover, extragalactic sources as Active Galactic Nuclei or Gamma-Ray Bursts are considered as good candidates to accelerate particles to such extreme energies. UHECRs are expected to travel from their sources through the extragalactic space and to interact with photon fields that fill it (Cosmic Microwave Background, CMB, and Extragalactic Background Light, EBL). These interactions, such as the photo-pion production and the photo-disintegration in the case of UHECR nuclei, would appear as low energy processes in the center of momentum. In contrast, in the laboratory frame very large Lorentz factors can be reached and the Special Relativity can be tested. The possibility of putting limits on LIV parameters with processes involving UHECRs was first discussed in [1]. Later on, the suppression of the flux at the highest energies was established and limits on LIV parameters were derived. As an example, parameter space studies were performed using the UHECR spectrum and a pure proton composition [2,3]. Motivated by experimental indications of chemical composition of UHECRs heavier than protons, the modifications of LI in the propagation of nuclei were studied for example in [4]. A detailed summary can be found in [5].

In this work LIVs are taken into account during the extragalactic propagation of UHECRs. A fit of the UHECR spectrum [6] and composition data [7] is performed as done in [8] and the results including the propagation in presence of LIV are discussed.

\section{LIV and UHECR propagation}

LI modifications can be implemented in many ways, with different consequences on physical observables. A simple approach consists in proposing a modified dispersion relation for particles and keeping the usual conservation of energy and momentum. LIV will affect the dispersion relation as

$$
E_{i}^{2}-p_{i}^{2}=m_{i}^{2} \Rightarrow \mu_{i}^{2}\left(E, p, M_{\mathrm{Pl}}\right) \approx m_{i}^{2}+\sum_{n=0}^{N} \eta_{i}^{(n)} \frac{E_{i}^{2+n}}{M_{\mathrm{Pl}}^{n}}
$$

where $p=|\vec{p}|, \mu$ is a function of momenta and energy, and $\eta_{i}$, which can be either positive or negative, parametrizes the strength of LIV for particle $i$. In the limit $\eta_{i}^{(n)}=0$ the Lorentz invariant dispersion relation is recovered. From Eq. 2.1 one can see that the LI correction becomes larger than the mass of the particles if $p \geq\left(m_{i}^{2} M_{\mathrm{Pl}}^{n} /\left|\eta_{i}^{(n)}\right|\right)^{1 /(2+n)}$. 


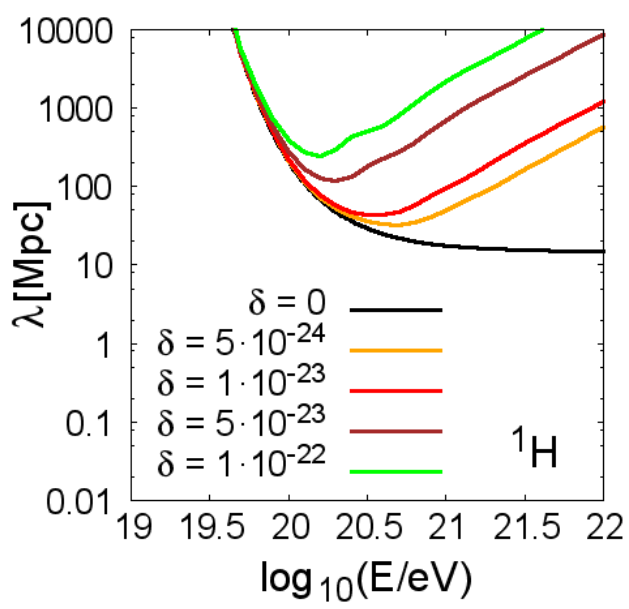

Figure 1: Attenuation length of protons interacting in the CMB at $z=0$ as a function of the proton energy in the laboratory frame, for the LI case (black line) and for different choices of the LIV coefficient $\delta$ for $n=0$ [9].

The LIV parameter can be defined as

$$
\delta_{i}^{(n)}=\frac{\eta_{i}^{(n)}}{M_{\mathrm{Pl}}^{n}}
$$

Here we adopt the LIV framework of [10], where a first order perturbation is considered. Since we consider $n=0$, the LIV parameter $\delta_{i}$ is dimensionless. It is interesting to notice that the LI violating term can be translated in a shift of the mass of the particle and a shift of the velocity from $c=1$. Since different particles can have different maximum attainable velocities, these can be different from $c$ as well as different from each other. The LIV parameter can be directly related to this difference [3].

We discuss first the photo-pion process. Soon after the discovery of the CMB it was realized that interactions of protons with CMB photons would deplete the CR flux at the highest energies ("GZK effect") [11, 12]. A suppression of the flux at the highest energies was actually measured [13, 14]. However, its origin is still unknown, since this feature is sensitive to local deficit of sources and can also be mimicked by acceleration cutoffs at the source. The inelasticity of the process, meaning how much of the energy of the initial proton is carried away by the final pion, is modified introducing LIV. The LIV affecting the photo-pion production gives the most important contribution to the modification of the UHECR spectrum above the GZK energy. By considering modifications to the maximum attainable velocity of the pion, the phase space allowed for the interaction is reduced with respect to the LI case. This is due to the limits on the allowed range of the interaction angles coming out from kinematics study [10]. The effect of different $\delta$ parameters on the attenuation length of the protons in the CMB is shown in Fig. 1, where it is clear that changing the value of $\delta$, the energy at which the LIV becomes significant is lower [9]. As a consequence, the UHECR spectrum is expected to exhibit a suppression near the GZK and to recover at higher energies.

Concerning the propagation of nuclei in the Universe, the energy losses for photo-pion production are shifted at energies $\sim A$ times higher than the threshold for protons. However, the excitation of the giant dipole resonance (GDR) by photons of energy tens of $\mathrm{MeV}$ in the nucleus rest frame 

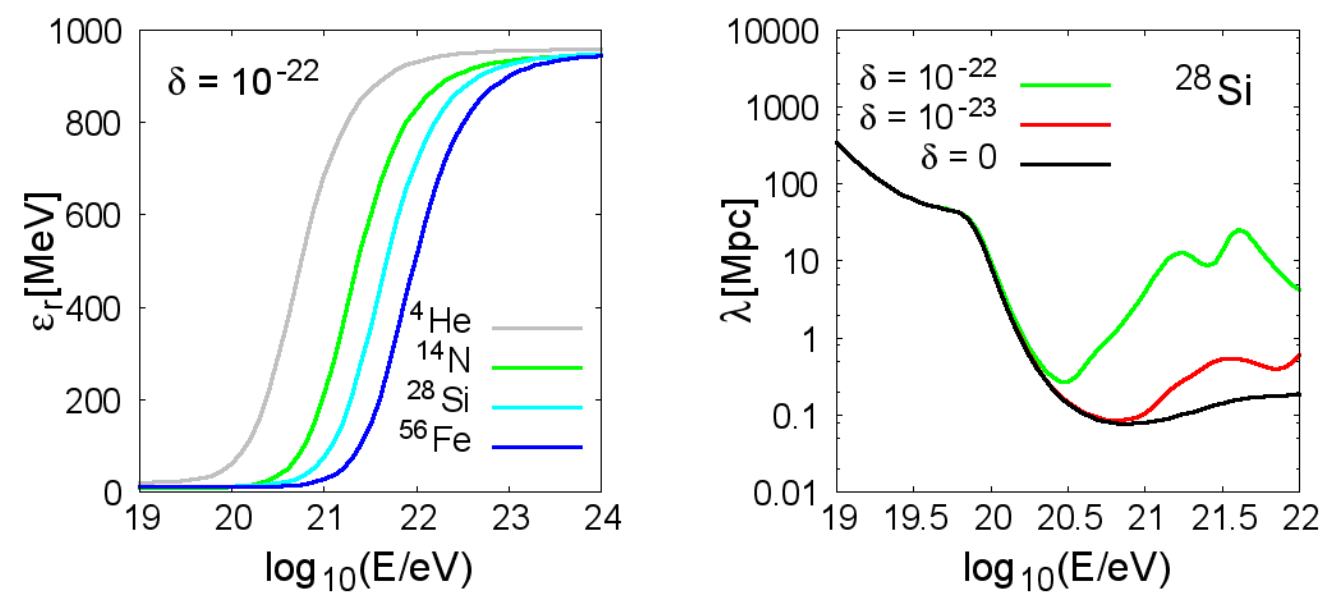

Figure 2: Left: Threshold energy in the nucleus rest frame for the photo-disintegration of nuclei as a function of the initial nucleus energy in the laboratory frame, for $\delta=10^{-22}$ [9]. Right: Mean free path for the photo-disintegration of an silicon nucleus as a function of the energy in the laboratory frame, at $z=0$. The LI scenario (black line) is compared to the LIV scenarios with $\delta=10^{-22}$ and $\delta=10^{-23}$ for $n=0$ [9].

causes the disintegration of the nucleus ejecting one or more nucleons and is responsible for the steepening of the flux at the highest energies. The dispersion relation for nuclei can be written assuming a superposition model for nuclei, i.e. considering them as the combination of $A$ nucleons of energy $E / A$. In this way, since we are dealing with the linear modifications $n=0$, the LIV parameter can be assumed as equal to the one for protons. The threshold energy of the photon in the nucleus rest frame does not depend on the energy of the nucleus but only on its nuclear mass; including LIV effects makes the dependence on the energy of the nucleus appear, as shown in Fig. 2 (right panel). The effect in the spectrum is expected to be similar to what happens to the proton case in presence of LIV, since also in this case the mean free path departs from the LI case and increases with energy, as shown in the right panel of Fig. 2 for silicon nuclei.

The discussed modifications to LI were included [9] in SimProp [15], a simulation code for the propagation of UHECRs in the extragalactic space. The physical quantities used in the code to take into account the EBL model and the photo-disintegration cross sections for this study are the Gilmore EBL model [16] and the Puget-Stecker-Bredekamp (PSB) model [17, 18] respectively.

\section{Fit results}

A fit of the cosmic ray spectrum [6] and composition data [7] as measured by the Pierre Auger Observatory is performed following the method described in [8]. The UHECR sources are assumed to be identical and homogeneously distributed in a co-moving volume. The mass species at the injection are: ${ }^{1} \mathrm{H},{ }^{4} \mathrm{He},{ }^{14} \mathrm{~N}$ and ${ }^{28} \mathrm{Si}$, being the inclusion of heavier masses not relevant for improving the goodnees of fit, as found in [8]. The injection spectrum is taken as:

$$
\frac{d N_{A}}{d E}=J_{A}(E)=f_{A} J_{0}\left(\frac{E}{10^{18} \mathrm{eV}}\right)^{-\gamma} \times f_{\text {cut }}\left(E, Z_{A} R_{\text {cut }}\right)
$$




\begin{tabular}{|l|c|c|c|c|c|c|c|c|c|}
\hline & $\gamma$ & $\log _{10}\left(R_{\text {cut }} / \mathrm{V}\right)$ & $\mathrm{H}(\%)$ & $\mathrm{He}(\%)$ & $\mathrm{N}(\%)$ & $\mathrm{Si}(\%)$ & $D(J)$ & $D\left(X_{\max }\right)$ & $D$ \\
\hline \hline LI, $\delta=0$ & 0.96 & 18.68 & 0. & 67.3 & 28.1 & 4.6 & 13.3 & 161.1 & 174.4 \\
LIV, $\delta=5 \cdot 10^{-24}$ & 0.91 & 18.65 & 0. & 71.8 & 23.9 & 4.3 & 15.1 & 163.5 & 178.5 \\
LIV $\delta=1 \cdot 10^{-23}$ & 0.91 & 18.65 & 0. & 71.4 & 24.3 & 4.3 & 14.9 & 163.6 & 178.5 \\
LIV $\delta=1 \cdot 10^{-22}$ & 0.94 & 18.65 & 0. & 72.8 & 22.7 & 4.6 & 18.2 & 163.6 & 181.8 \\
$\max$ LIV & 0.95 & 18.40 & 62.3 & 32.2 & 5.4 & 0.08 & 27.3 & 162.0 & 189.3 \\
\hline
\end{tabular}

Table 1: Best-fit parameters for the LI reference model in [8] and for the LIV cases with different $\delta$ values. The maximal-LIV case is also reported. The fractions are defined at fixed energy $\left(E=10^{18} \mathrm{eV}\right)$.

where $f_{A}$ is the fraction of the injected isotope over the total and is defined at fixed energy $\left(E=10^{18}\right.$ $\mathrm{eV})$. The cutoff function is:

$$
f_{\text {cut }}\left(E, Z_{A} R_{\text {cut }}\right)= \begin{cases}1 & \left(E<Z_{A} R_{\text {cut }}\right) \\ \exp \left(1-\frac{E}{Z_{A} R_{\text {cut }}}\right) & \left(E>Z_{A} R_{\text {cut }}\right)\end{cases}
$$

The parameters of the fit are the spectral index $\gamma$, the cutoff rigidity $R_{\text {cut }}$, the normalization of the spectrum $J_{0}$ and three of the fractions $f_{A}$, the fourth being fixed by $\sum_{A} f_{A}=1$.

For the spectrum we fit the surface detector (SD) event distribution using a forward-folding procedure. For the composition we fit the $X_{\max }$ distribution adopting a Gumbel parametrization [19]. Both the spectrum and composition are fitted at energies $\log _{10}(E / \mathrm{eV})>18.7$, i.e. above the ankle. In the Auger data the energy spectrum and the $X_{\max }$ distributions are independent measurements and the model likelihood is therefore given by $L=L_{J} \cdot L_{X_{\max }}$. The goodness-of-fit is assessed with a generalized $\chi^{2}$, (the deviance, D), defined as the negative log-likelihood ratio of a given model and the saturated model that perfectly describes the data:

$$
D=D(J)+D\left(X_{\max }\right)=-2 \ln \frac{L}{L^{\text {sat }}}=-2 \ln \frac{L_{J}}{L_{J}^{\text {sat }}}-2 \ln \frac{L_{X_{\max }}}{L_{X_{\max }}^{\text {sat }}}
$$

To account for the LIV effects in the propagation of protons and nuclei we use the modified version [9] of SimProp, as earlier anticipated. The LIV parameter $\delta$ is considered to be the same in the photo-pion and photo-disintegration process. The simulations are performed for different $\delta$ values and the corresponding best-fit parameters are reported in Table 1, where the corresponding LI case and the maximal-LIV cases are compared. The best-fit parameters are found to be very similar to the LI case: the spectral index is hard and the rigidity cutoff is low, in order to reproduce the low level of mixture of masses at each energy.

Since the effect of enhancing the LIV parameter is to increase the interaction length of the particles, a way to investigate an extreme case is to switch off all the interactions with background photons [20]. The maximal-LIV case is simulated with a simplified version of the propagation code, where only the adiabatical energy losses due to the expansion of the Universe are taken into account. The corresponding observables are shown in Fig. 3. The spectral index at the best fit is similar to the LI and partial LIV cases. A remarkable difference is visible in the rigidity at the best fit: in this case the rigidity has to be lower at the source with respect to the other case in order to compensate for the absence of interactions that would lower it during propagation. Another substantial difference 

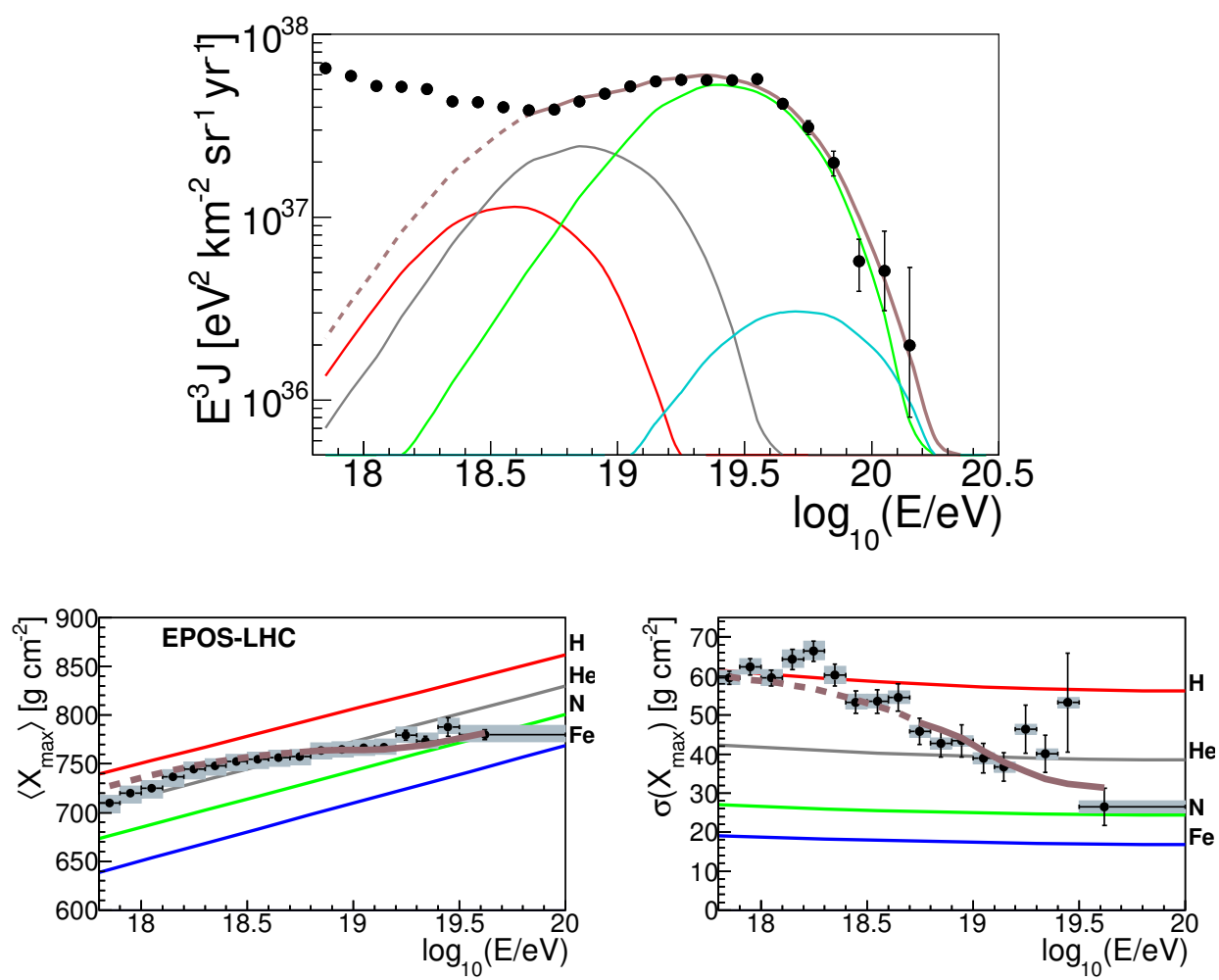

Figure 3: Top: simulated energy spectrum of UHECRs (multiplied by $E^{3}$ ) at the top of the Earth's atmosphere, obtained with the best-fit parameters for the case with maximal-LIV. Spectra at Earth are grouped according to the mass number as follows: $A=1$ (red), $2 \leq A \leq 4$ (grey), $5 \leq A \leq 22$ (green), $23 \leq A \leq 28$ (cyan), total (brown), compared to data from [6]. Bottom: average and standard deviation of the $X_{\max }$ distribution as predicted (assuming EPOS-LHC [21] for UHECR-air interactions) for the model versus pure $\left({ }^{1} \mathrm{H}\right.$ (red), ${ }^{4} \mathrm{He}$ (grey), ${ }^{14} \mathrm{~N}$ (green) and ${ }^{56} \mathrm{Fe}$ (blue)) compositions, compared to data from [22]. Only the energy range where the brown lines are solid is included in the fit.

is that in the LI or partial LIV cases the proton fraction at the source is almost negligible, while in the maximal-LIV case protons must be present already at the source in order to compensate for the absence of interactions.

\section{Discussion and conclusions}

In this work we use the interactions of UHECRs during their propagation from the sources to the Earth in order to investigate the possible violations of LI. A combined fit of the spectrum and composition data is used here for the first time for this purpose. In the LI case, it is shown that a scenario including a hard spectral index and a low rigidity cutoff can describe the data [8]. As a consequence, at the energies corresponding to the rigidity cutoff found from the fit, the LI corrections are not larger than the mass of the considered nuclei and the partial LIV cases are not distinguishable from the LI case. This can be also shown in Fig. 4, where the positions of the best-fit cases are found at very similar values (around $\gamma \sim 1$ ). 


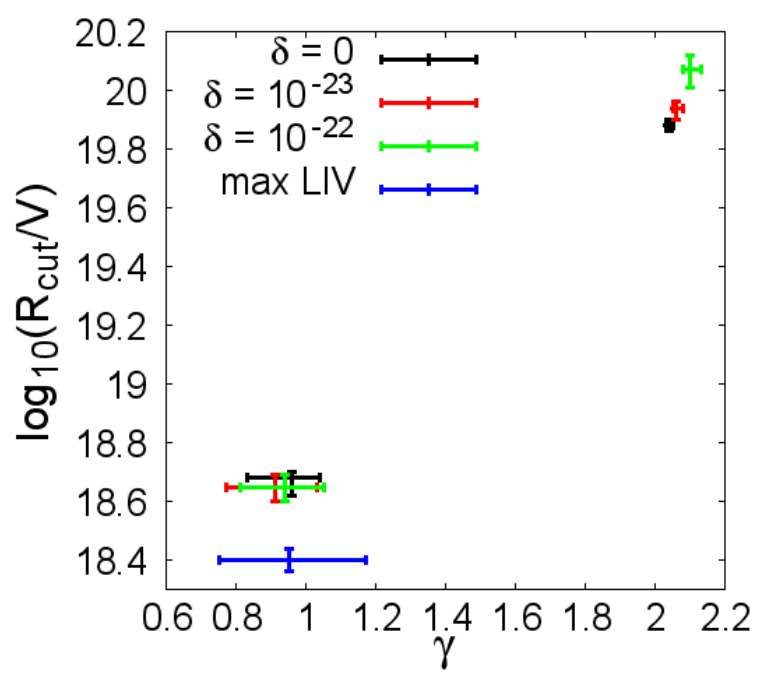

Figure 4: Position of the best-fit values as a function of the spectral index and rigidity cutoff, for the LI case and some of the LIV cases, are reported in the lower-left part of the figure. The error bars are computed from the interval $D \leq D_{\min }+1$. The local minima are reported in the upper-right part of the plot: in this case the error bars are computed from the interval $D \leq D_{\min }^{\text {local }}+1$.
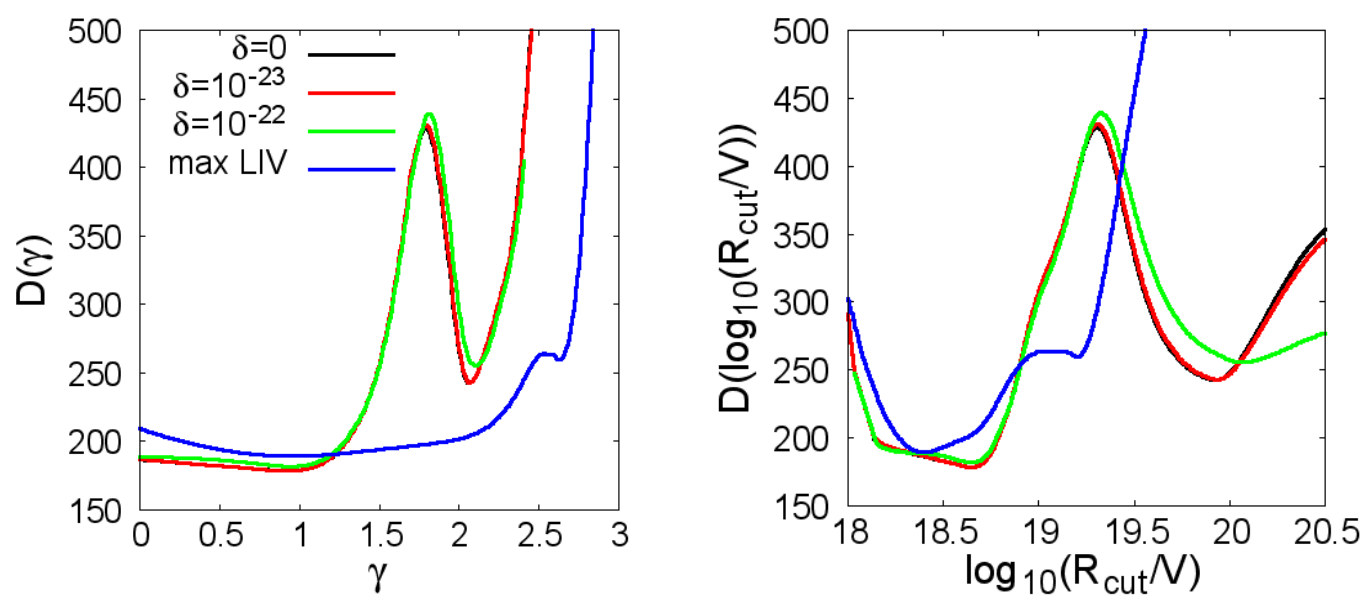

Figure 5: Deviance $D$ versus $\gamma$ (left) and $R_{\text {cut }}$ (right) for the LI and LIV cases.

We also find a local minimum region around $\gamma \sim 2$ and $\log _{10}\left(R_{\text {cut }} / \mathrm{V}\right) \sim 20$, as it is shown in Fig. 4. At the energies corresponding to these values of the rigidity, we start to be sensitive to the modifications to LI, mainly due to the survival of the light mass component up to the highest energies. A trend towards higher rigidities and softer spectral indices is visible while increasing the value of the LIV parameter. This is motivated by the fact that a larger $\delta$ implies that the interaction length starts to increase at lower energies (see Fig. 2, right panel). As a result, the allowed rigidity cutoff at the source is larger. However, by looking at Fig. 5, where the deviance versus the spectral index and the rigidity cutoff is reported, it is clear that the significance of the local minimum is much smaller with respect to the main one, making this trend not useful for drawing a conclusion about the possibility of disfavoring a LIV parameter with the current measurements, which prefer a low- 
rigidity scenario.

We have also investigated an extreme case in which the interactions are switched off: this case can be reported to modifications of LI having $\eta<-2.5 \times 10^{-14}$ for $n=1$ or $\eta<-4 \times 10^{-7}$ for $n=2$, as studied in [20]. By comparing the values of the deviance at the minimum (see Table 1 ), the maximal-LIV case can be disfavored at more than the $3 \sigma$ level. However, the absence of the interactions makes the maximal-LIV case more sensitive to the initial conditions, namely the number of injected species at the source and/or the choice for the functional shape of the cutoff at the sources.

\section{References}

[1] R. Aloisio et al., Phys. Rev. D 62, 053010 (2000) [astro-ph/0001258].

[2] X. J. Bi et al., Phys. Rev. D 79, 083015 (2009) [arXiv:0812.0121 [astro-ph]].

[3] S. T. Scully and F. W. Stecker, Astropart. Phys. 31, 220 (2009) [arXiv:0811.2230 [astro-ph]].

[4] A. Saveliev et al., JCAP 1103, 046 (2011) [arXiv:1101.2903 [astro-ph.HE]].

[5] S. Liberati, Class. Quant. Grav. 30, 133001 (2013) [arXiv:1304.5795 [gr-qc]].

[6] I. Valiño for the Pierre Auger Collaboration, PoS(ICRC2015)271, [arXiv:1509.03732].

[7] A. Aab et al. [Pierre Auger Collaboration], Phys. Rev. D 90, no. 12, 122005 (2014), [arXiv:1409.4809].

[8] A. Aab et al. [Pierre Auger Collaboration], JCAP 1704, no. 04, 038 (2017), [arXiv:1612.07155].

[9] R. Guedes Lang, Master Thesis, University of São Paulo, Brazil (2017).

[10] S. R. Coleman and S. L. Glashow, Phys. Rev. D 59, 116008 (1999) [hep-ph/9812418].

[11] K. Greisen, Phys. Rev. Lett. 16, 748 (1966).

[12] G. T. Zatsepin and V. A. Kuzmin, JETP Lett. 4, 78 (1966)

[13] J. Abraham et al. [Pierre Auger Collaboration], Phys. Rev. Lett. 101, 061101 (2008) [arXiv:0806.4302 [astro-ph]].

[14] R. U. Abbasi et al. [HiRes Collaboration], Phys. Rev. Lett. 100, 101101 (2008) [astro-ph/0703099].

[15] R. Aloisio et al., JCAP 1210, 007 (2012), [arXiv:1204.2970].

[16] R. C. Gilmore et al., Mon. Not. Roy. Astron. Soc. 422, 3189 (2012) [arXiv:1104.0671 [astro-ph.CO]].

[17] J. L. Puget et al., Astrophys. J. 205, 638 (1976).

[18] F. W. Stecker and M. H. Salamon, Astrophys. J. 512, 521 (1999) [astro-ph/9808110].

[19] M. De Domenico et al., JCAP 1307, 050 (2013) [arXiv:1305.2331 [hep-ph]].

[20] D. Boncioli et al., PoS(ICRC2015)521 [arXiv:1509.01046 [astro-ph.HE]].

[21] T. Pierog et al., Phys. Rev. C 92, no. 3, 034906 (2015) [arXiv:1306.0121 [hep-ph]].

[22] A. Porcelli for the Pierre Auger Collaboration, PoS(ICRC2015)420, [arXiv:1509.03732]. 